\title{
Analysis of Anticoagulant Effect of Unfractionated Heparin by Using Thrombin Generation Assay in Vitro
}

\author{
Atsuki Yamashita ${ }^{1}$, Chiai Nagae ${ }^{1}$, Mika Mori $^{1}$, Tomoko Ashikaga ${ }^{2}$, \\ Mieko Akita ${ }^{1}$, Noriko Suzuki ${ }^{3}$, Satoshi Yamazaki ${ }^{3}$, Shinobu Tatsunami ${ }^{4}$, \\ Shigenobu Takayama ${ }^{5}$, and Masashi Taki ${ }^{2}$
}

(Received for Publication: November 18, 2014)

\begin{abstract}
Unfractionated heparin (UFH) is widely used in the treatment and prophylaxis of thrombosis. The anticoagulant effect of UFH is monitored according to activated partial thromboplastin time (APTT). However, there are reports of cases in which hemorrhagic complications develop despite APTT being maintained within the therapeutic range. This indicates there are problems with the monitoring method using APTT during UFH treatment. To assess the actual anticoagulant effect in UFH therapy using APTT as a monitoring method, we investigated changes in APTT and thrombin generation (TG) potential in vitro with the addition of UFH to samples from 10 healthy adults. There were large individual differences in the degree of APTT prolongation with the addition of UFH to samples from healthy adults. Furthermore, individual differences in the degree of TG change exceeded those in APTT in 7 subjects with comparable APTT changes caused by UFH (coefficients of variation: 2-5\% for APTT, 10-50\% for TG potential). Thus, with UFH treatment, there are large individual differences in the degree of TG inhibition even when APTT is within the appropriate range for coagulation control. These findings provide invaluable information for resolving the problem of hemorrhagic complications during UFH treatment based on the current APTT method.
\end{abstract}

\section{Key words}

unfractionated heparin, anticoagulant effect, thrombin generation, activated partial thromboplastin time, individual difference

\section{Introduction}

Unfractionated heparin (UFH) is widely used in the treatment and prophylaxis of thrombosis. Doses are adjusted to ensure the prolongation of activated partial thromboplastin time (APTT) is within 1.5-2.5 times the normal value when UFH is used for treatment ${ }^{1}$. However, it has been reported that there are large individual differences in the APTT-prolongation effect of UFH, and how precisely APTT reflects the actual anticoagulation effect of UFH in vivo is unknown $^{2-4)}$. Furthermore, the effect of UFH on APTT measurement varies markedly depending on the reagents and/or measurement equipment used ${ }^{5-9}$. Thus, there are problems with using APTT as a monitoring method in UFH treatment. The incidence of hemorrhagic complications when UFH treatment is performed with APTT monitoring is reportedly 2$5 \%{ }^{10) 11}$, and there are cases in which hemorrhagic complications occur despite APTT being maintained

\footnotetext{
1 Department of Pediatrics, St. Marianna University School of Medicine

2 Department of Pediatrics, St. Marianna University School of Medicine, Yokohama City Seibu Hospital

3 Department of Clinical Laboratory, St. Marianna University School of Medicine Hospital

4 Department of Medical Statistics, St. Marianna University School of Medicine

5 Faculty of Health Science, Daito Bunka University
} 
within the therapeutic range. In these cases, the actual anticoagulant effect of UFH may be excessive, despite appropriate APTT prolongation.

Thrombin is well known to play an important role in coagulation mechanisms. Measuring the amount of thrombin generated more precisely reflects overall hemostasis than coagulation times, such as prothrombin time (PT) and APTT. The thrombin generation (TG) test was recently proposed to be an informative method for studying the anticoagulant effect of antithrombotic drugs ${ }^{12) 13)}$.

Therefore, in order to assess the anticoagulant effect of UFH therapy using APTT as a monitoring method, this study investigated changes in APTT and TG potential in response to the addition of UFH using the TG test according to the calibrated automated thrombogram method.

\section{Methods}

\section{Blood Samples}

Ten healthy adults (6 men and 4 women) aged 20-40 years with normal PT and APTT served as the study subjects. Whole blood was drawn into citrated tubes. Platelet-poor plasma (PPP) was prepared by 2 centrifugations at $3000 \mathrm{rpm}$ for $15 \mathrm{~min}$ at $15^{\circ} \mathrm{C}$; it was subsequently aliquotted and stored at $-80^{\circ} \mathrm{C}$ until use.

This study was approved by the Ethics Committee at St. Marianna University School of Medicine (approval \#1210), and informed consent for blood collection was directly obtained from all subjects.

\section{Heparin}

The UFH preparation used was the Heparin $\mathrm{Na}$ Lock (1000 U/10 mL) Syringe ${ }^{\circledR}$ (Otsuka Pharmaceutical, Tokyo, Japan). Heparin was diluted with saline, $20 \mu \mathrm{L}$ heparin solution was added to $980 \mu \mathrm{L}$ plasma, and the final concentration was adjusted to $0.1,0.2$, $0.3,0.4$, and $0.5 \mathrm{U} / \mathrm{mL}$. In addition, $20 \mu \mathrm{L}$ saline was added to $980 \mu \mathrm{L}$ plasma to create $0 \mathrm{U} / \mathrm{mL}$ sample.

\section{Determination of APTT}

UFH concentration $(0,0.2,0.3,0.4$, and 0.5 $\mathrm{U} / \mathrm{mL}$ ) was added to PPP obtained from 10 healthy adults, and changes in coagulation time were evaluated by the APTT method. APTT was measured using a CA-7000 ${ }^{\circledR}$ analyzer (Sysmex, Kobe, Japan), and APTT-SLA $^{\circledast}$ (Sysmex Corporation) was used as an APTT reagent.

\section{TG Assay}

UFH $(0,0.1,0.2$, and $0.3 \mathrm{U} / \mathrm{mL})$ was added to PPP obtained from 7 healthy adults in whom APTT changes as a result of UFH addition were comparable, and changes in TG potential were evaluated. TG potential was measured using Fluoroskan Ascent ${ }^{\circledR}$ (Finggal Link, Tokyo, Japan) and analyzed using the Thrombinoscope ${ }^{\circledR}$ Program (Finggal Link). PPP Reagent (5 pM tissue factor and $4 \mu \mathrm{M}$ phospholipid) and Flu-Ca kit $(16.6 \mathrm{mM} \mathrm{CaCl} 2)$ were used as reagents (Finggal link). Prepared plasma samples were incubated at $37^{\circ} \mathrm{C}$ for $5 \mathrm{~min}$ until immediately before measurement. TG potential was evaluated using the following 5 parameters: lag time, thrombin peak (Peak), endogenous thrombin potential (ETP), time to peak (ttPeak), and start tail.

\section{Statistical Method}

For analyzing correlations of APTT with TG parameters when UFH was added to samples, Spearman's rank correlation test was used for statistical analysis. P-values $<0.05$ were considered to be statistically significant.

\section{Results}

\section{APTT}

The concentration at which APTT was prolonged at least 1.5 -fold was $0.2 \mathrm{U} / \mathrm{mL}$ in 7 of the 10 subjects and $0.3 \mathrm{U} / \mathrm{mL}$ in the other 3 . Individual differences in APTT changes became significant with increasing UFH concentration; APTT was prolonged at slightly more than 2-fold with the addition of 0.5 $\mathrm{U} / \mathrm{mL}$ UFH in the subject with the smallest APTT change, while APTT was prolonged approximately 6-fold in another subject with the same concentration of UFH (Figure 1). The antithrombin (AT) level of the subject with the smallest APTT change was within the normal range (data not shown).

\section{TG test}

TG potential was evaluated in 7 subjects in whom APTT prolongation as a result of UFH was comparable. APTT was prolonged 1.5-2.5-fold with the addition of 0.2 or $0.3 \mathrm{U} / \mathrm{mL}$ UFH (Figure 2).

The thrombin generation curve (thrombogram) shifted to the right with the addition of UFH, the peak decreased, and the amount of thrombin generated was inhibited in a dose-dependent manner (Figure 3). Regarding the TG parameters, ETP and Peak decreased, while lag time, ttPeak, and start tail were prolonged with the addition of UFH (Figure 4). 


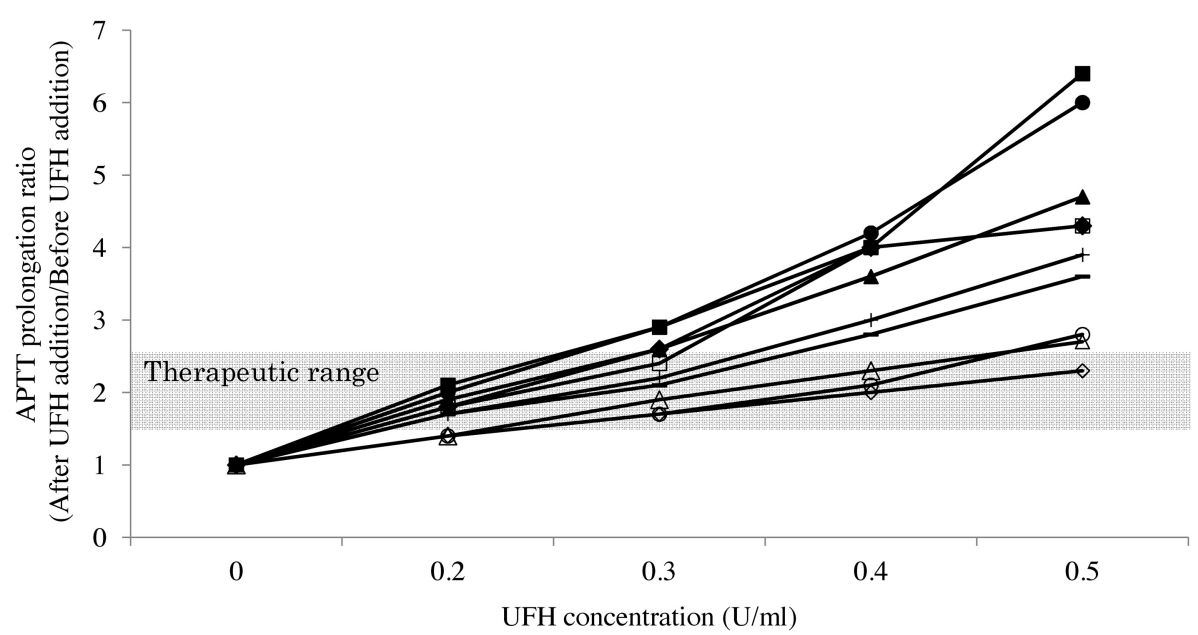

Figure 1. APTT prolongation with in vitro addition of UFH in samples from 10 healthy adults.

The UFH concentration at which APTT prolongation became at least 1.5-fold was 0.2

$\mathrm{U} / \mathrm{mL}$ in 7 of the 10 subjects and $0.3 \mathrm{U} / \mathrm{mL}$ in the other 3 subjects.

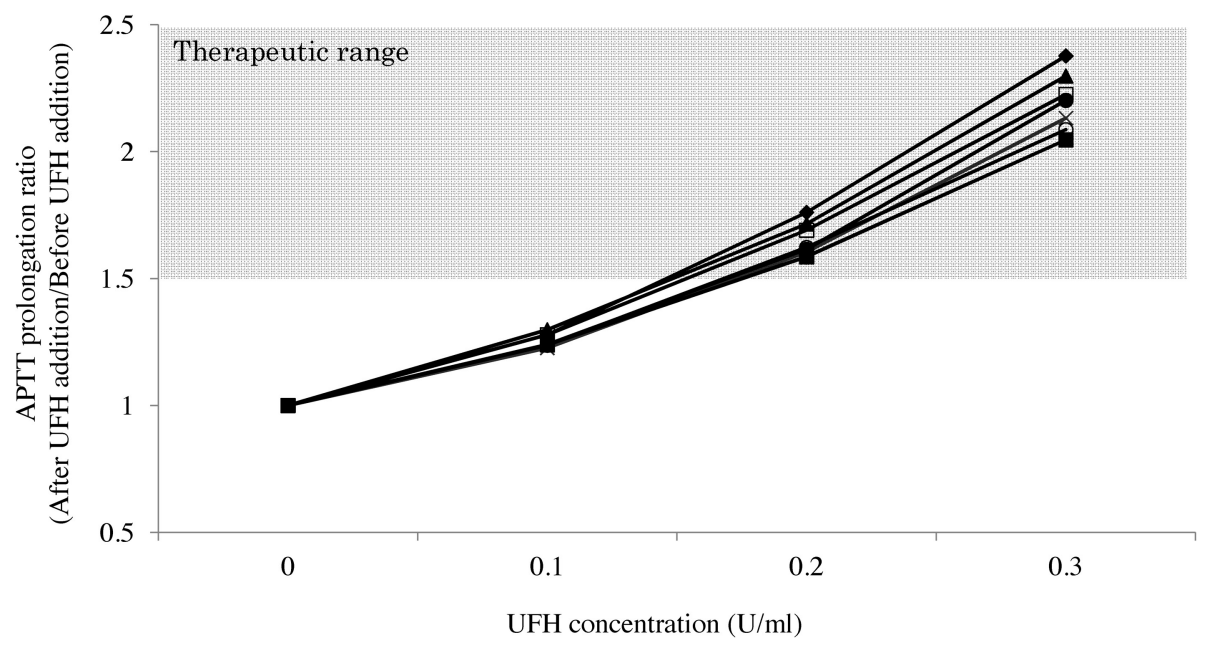

Figure 2. APTT prolongation with the addition of UFH to samples from 7 healthy adults in whom clotting times were equally prolonged by UFH.

APTT prolongation became at least 1.5-fold in all subjects with the addition of UFH $0.2 \mathrm{U} / \mathrm{mL}$, and prolongations with the addition of $0.3 \mathrm{U} / \mathrm{mL}$ were within the range of $2-$ to 2.5 -fold in all subjects.

These results indicate TG potential is inhibited by the addition of UFH in a dose-dependent manner.

In 7 subjects with comparable UFH sensitivity measured by the APTT method, APTT was negatively correlated with ETP $(r=-0.86, p<0.01)$ and Peak $(r=-0.77, p<0.01)$ and positively correlated with lag time $(r=0.85, p<0.01)$, ttPeak $(r=0.94$, $p<0.01)$, and start tail $(r=0.90, p<0.01)$; however, large prediction intervals were detected in correlation with APTT and any TG parameters (Figure 5). When the change ratios (calculated as after UFH addition / before UFH addition) in APTT and TG parameters were compared using coefficient of variations $(\mathrm{CVs})$, the CV of APTT was very small at $2-5 \%$, while those of ETP and Peak were 10-46\% and 39-50\%, respectively; meanwhile, the CVs of lag time, ttPeak, and start tail, which are the TG parameters reflecting time, were relatively low (Table 1). 


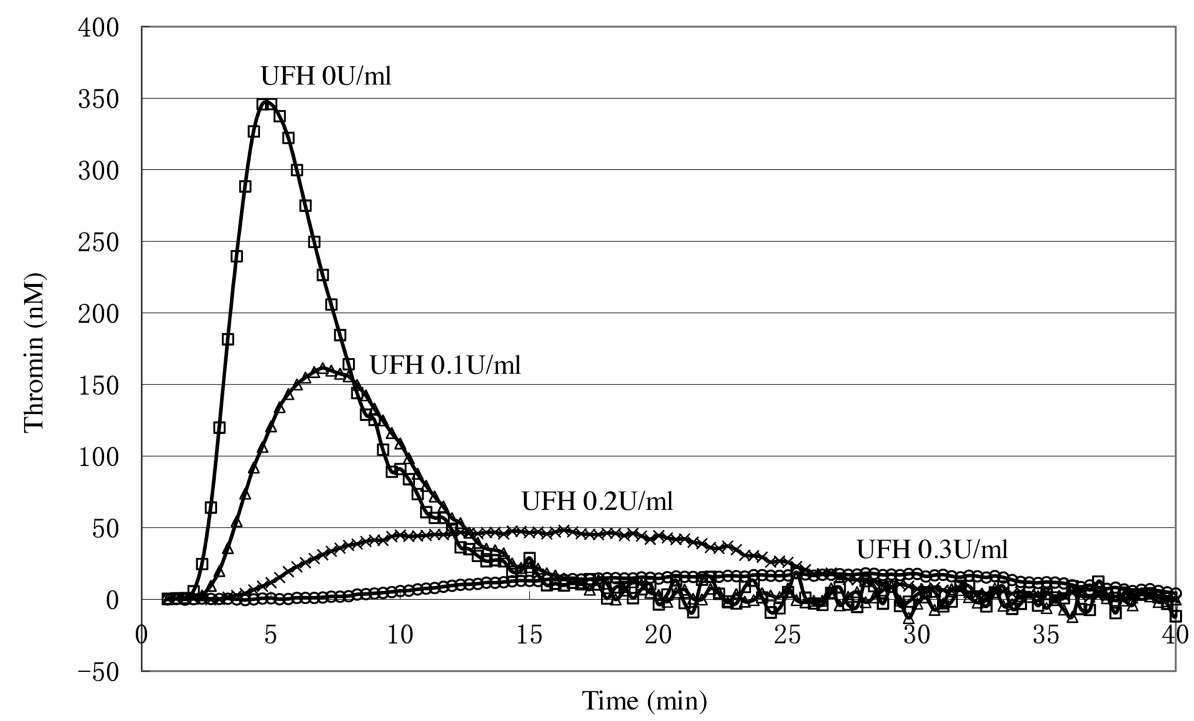

Figure 3. Dose-dependent inhibition of UFH $(0,0.1,0.2$, and $0.3 \mathrm{U} / \mathrm{mL})$ on $\mathrm{TG}$ test in a healthy adult.

Each thrombin generation curve (thrombogram) was shown in the presence of UFH $0 \mathrm{U} / \mathrm{ml}(\square)$, UFH $0.1 \mathrm{U} / \mathrm{ml}(\triangle)$, UFH $0.2 \mathrm{U} / \mathrm{ml}(\times)$, and UFH $0.3 \mathrm{U} / \mathrm{ml}(\bigcirc)$, respectively.
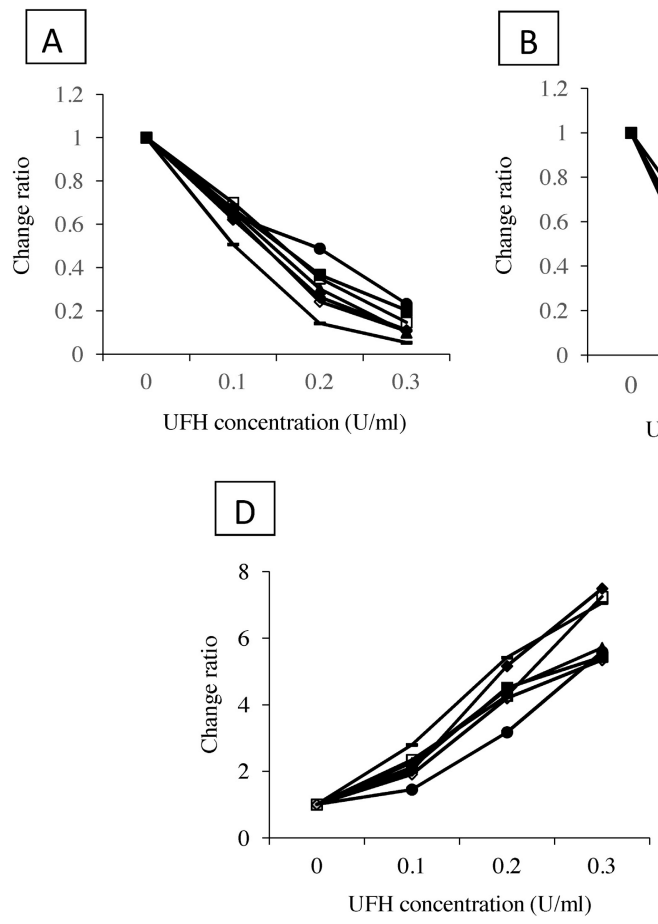
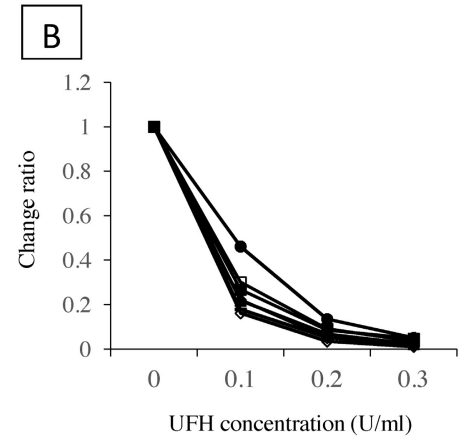
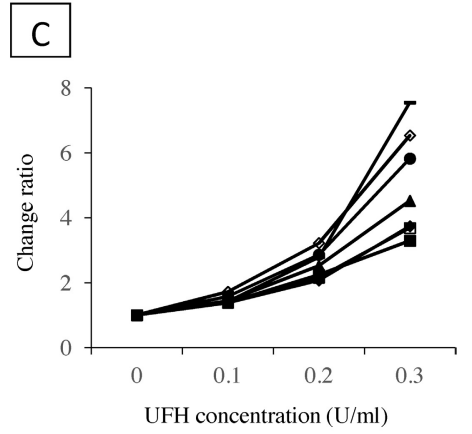

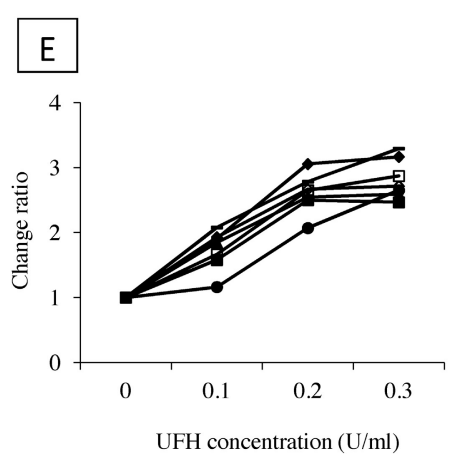

Figure 4. The alteration of each TG parameters by addition of UFH in 7 healthy adults with comparable APTT changes when several UFH concentrations $(0$ to $0.3 \mathrm{U} / \mathrm{mL})$ were added in vitro.

ETP (A) and Peak (B) decreased, while lag time (C), ttPeak (D), and start tail (E) were prolonged with the addition of UFH. 


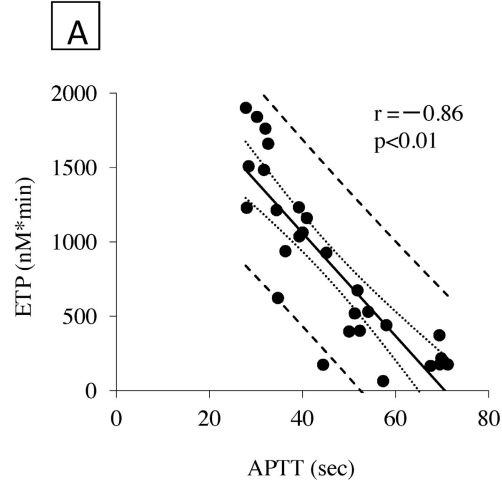

D
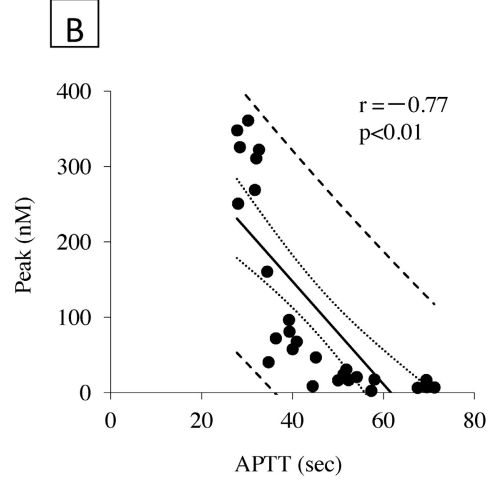
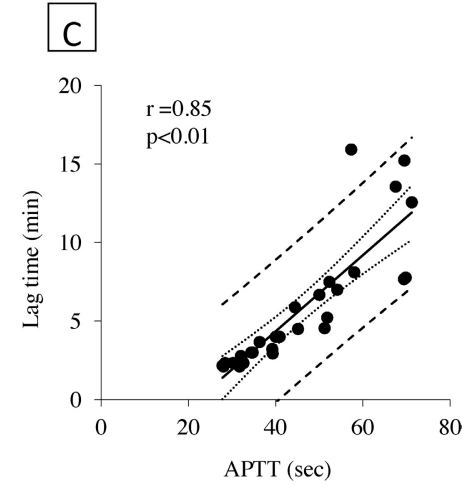

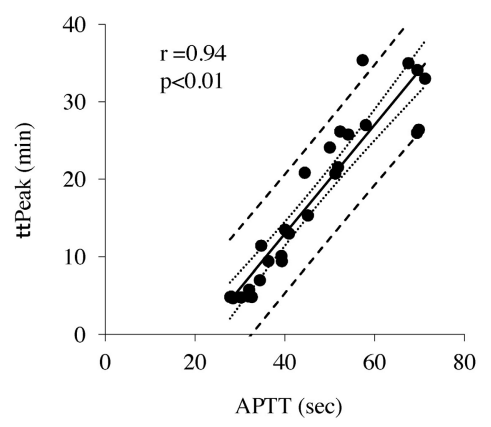

$\mathrm{E}$

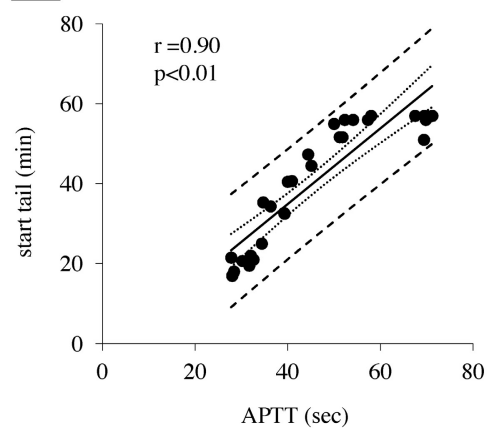

Figure 5. Correlations of APTT with TG parameters in 7 healthy adults with comparable APTT changes when several UFH concentrations ( 0 to $0.3 \mathrm{U} / \mathrm{mL}$ ) were added in vitro.

Spearman's rank correlation test was used for statistical analysis. The solid line represents regression line, dot lines represent 95\% confidence interval, and dashed lines represent 95\% prediction interval. APTT was negatively correlated with ETP (A) and Peak (B), while it was positively correlated with lag time (C), ttPeak (D), and start tail (E). However, large prediction intervals were detected in correlation with APTT and any TG parameters.

Table 1. Change Ratio in TG Parameters with the Addition of UFH to Samples from 7 Healthy Adults with Comparable APTT Changes

\begin{tabular}{|c|c|c|c|c|c|c|}
\hline \multirow{3}{*}{ Parameters } & \multicolumn{6}{|c|}{$\mathrm{UFH}(\mathrm{U} / \mathrm{ml})$} \\
\hline & \multicolumn{2}{|l|}{0.1} & \multicolumn{2}{|l|}{0.2} & \multicolumn{2}{|l|}{0.3} \\
\hline & $\begin{array}{c}\text { Change ratio } \\
(\text { Mean } \pm \mathrm{SD})\end{array}$ & $\begin{array}{l}\mathrm{CV} \\
(\%)\end{array}$ & $\begin{array}{l}\text { Change ratio } \\
(\text { Mean } \pm \mathrm{SD})\end{array}$ & $\begin{array}{l}\mathrm{CV} \\
(\%)\end{array}$ & $\begin{array}{l}\text { Change ratio } \\
(\text { Mean } \pm \mathrm{SD})\end{array}$ & $\begin{array}{l}\mathrm{CV} \\
(\%)\end{array}$ \\
\hline APTT & $1.26 \pm 0.03$ & 2.2 & $1.66 \pm 0.07$ & 4 & $2.20 \pm 0.12$ & 5.3 \\
\hline ETP & $0.63 \pm 0.06$ & 9.7 & $0.31 \pm 0.11$ & 35 & $0.14 \pm 0.06$ & 46 \\
\hline Peak & $0.26 \pm 0.10$ & 39 & $0.07 \pm 0.03$ & 46 & $0.03 \pm 0.01$ & 50 \\
\hline Lag time & $1.47 \pm 0.13$ & 8.6 & $2.55 \pm 0.42$ & 17 & $5.01 \pm 1.63$ & 33 \\
\hline ttPeak & $2.13 \pm 0.41$ & 19 & $4.46 \pm 0.73$ & 16 & $6.27 \pm 0.95$ & 15 \\
\hline Start tail & $1.74 \pm 0.30$ & 17 & $2.61 \pm 0.30$ & 12 & $2.82 \pm 0.31$ & 11 \\
\hline
\end{tabular}

$\mathrm{CV}$ : Coefficient of variance 


\section{Discussion}

Although low molecular weight heparin and factor Xa inhibitors have recently been developed for the treatment and prophylaxis of thrombosis, UFH is still the most widely used anticoagulant agent. UFH deactivates coagulation factors II, IX, X, XI, and XII via AT. Its anticoagulant effect is known to differ among individuals because of the multiple underlying anticoagulant mechanisms ${ }^{14)}$. The present investigation demonstrates that there are large individual differences in the degree of APTT prolongation with the addition of UFH in vitro. Furthermore, the anticoagulant effect of UFH is known to be less effective in subjects with low AT levels $(<60 \%)$ than in subjects with normal AT levels ${ }^{15)}$. In the present study, the AT level of the subject with the smallest APTT prolongation as a result of UFH addition was within the normal range.

In subjects with comparable APTT changes in response to UFH, each TG parameter obtained in the presence of UFH was strongly correlated with APTT values, demonstrating TG inhibition in a UFH concentration-dependent manner. However, individual differences in the change ratios of each TG parameter were larger than those with APTT, especially for ETP and Peak. These results suggest the degree of TG inhibition differs among individuals, even if APTT prolongation is equivalent. This is considered to be because strong inhibition of TG potential as a result of UFH may tend to be associated with hemorrhage; conversely mild TG inhibition may tend to be related to an insufficient anticoagulant effect. Individual differences in TG inhibition as a result of UFH addition may be the reason for the occurrence of hemorrhagic complications despite APTT being maintained within the therapeutic range during UFH treatment.

\section{Conclusion}

The degree of TG inhibition is associated with large individual differences, even when APTT is maintained within the appropriate range during UFH treatment. These findings provide invaluable information for resolving the problem of hemorrhagic complications during UFH treatment based on the current APTT method. This issue awaits clarification in further investigations using clinical samples.

\section{Disclosure of Conflicts of Interest}

The authors have no potential conflicts of interest to disclose.

\section{Acknowledgment}

A portion of this study was conducted using the "Preparation of Treatment Control Manual and Development of Training Method for the Improvement and Treatment Uniformity of Pediatric Hemorrhage and Thrombotic Disease" by the Research and Development Project of Child Health and Development.

\section{References}

1) Monagle P, Chalmers E, Chan A, DeVeber G, Kirkham F, Massicotte P, Michelson AD; American College of Chest Physicians. Antithrombotic therapy in neonates and children: American College of Chest Physicians EvidenceBased Clinical Practice Guidelines (8th Edition). Chest 2008; 133: 887-968.

2) Young E, Prins M, Levine MN, Hirsh J. Heparin binding to plasma proteins, an important mechanism for heparin resistance. Thromb Haemost 1992; 67: 639-643.

3) Hirsh J, van Aken WG, Gallus AS, Dollery CT, Cade JF, Yung WL. Heparin kinetics in venous thrombosis and pulmonary embolism. Circulation 1976; 53: 691-695.

4) Murray D, Pennell B, Olson J. Variability of prothrombin time and activated partial thromboplastin time in the diagnosis of increased surgical bleeding. Transfusion 1999; 39: 56-62.

5) Kitchen S, Jennings I, Woods TA, Preston FE. Wide variability in the sensitivity of APTT reagents for monitoring of heparin dosage. J Clin Pathol 1996; 49: 10-14.

6) Bjornsson TD, Nash PV. Variability in heparin sensitivity of APTT reagents. Am J Clin Pathol 1986; 86: 199-204.

7) Greaves M. Limitations of the laboratory monitoring of heparin therapy. Scientific and Standardization Committee Communications: on behalf of the Control of Anticoagulation Subcommittee of the Scientific and Standardization Committee of the International Society of Thrombosis and Haemostasis. Thromb Haemost 2002; 87: 163-164.

8) van den Besselaar AM, Meeuwisse-Braun J, Strebus A, Schaefer-van Mansfeld H, Witteveen E, van der Meer FJ. Response of the activated partial thromboplastin time (APTT) to heparin is influenced by coagulometers. Thromb Haemost 1995; 74: 1383-1384.

9) Smythe MA, Koerber JM, Westley SJ, Nowak 
SN, Begle RL, Balasubramaniam M, Mattson JC. Use of the activated partial thromboplastin time for heparin monitoring. Am J Clin Pathol 2001; 115: 148-155.

10) The Columbus Investigators: Low-molecularweight heparin in the treatment of patients with venous thromboembolism. N Engl J Med 1997; 337: 657-662.

11) Merli G, Spiro TE, Olsson CG, Abildgaard U, Davidson BL, Eldor A, Elias D, Grigg A, Musset D, Rodgers GM, Trowbridge AA, Yusen RD, Zawilska K; Enoxaparin Clinical Trial Group. Subcutaneous enoxaparin once or twice daily compared with intravenous unfractionated heparin for treatment of venous thromboembolic disease. Ann Intern Med 2001; 134: 191-202.

12) Robert S, Ghiotto J, Pirotte B, David JL, Masereel B, Pochet L, Dogné JM. Is thrombin generation the new rapid, reliable and relevant pharmacological tool for the development of anticoagulant drugs? Pharmacol Res 2009; 59: 160-166.

13) Al Dieri R, Alban S, Béguin S, Hemker HC. Thrombin generation for the control of heparin treatment, comparison with the activated partial thromboplastin time. J Thromb Haemost 2004; 2: 1395-1401.

14) Hirsh J, Warkentin TE, Shaughnessy SG, Anand SS, Halperin JL, Raschke R, Granger C, Ohman EM, Dalen JE. Heparin and low-molecular-weight heparin: mechanisms of action, pharmacokinetics, dosing, monitoring, efficacy, and safety. Chest 2001; 119: 64S-94S.

15) Despotis GJ, Levine V, Joist JH, Joiner-Maier D, Spitznagel E. Antithrombin III during cardiac surgery: effect on response of activated clothing time to heparin andrelationship to markers of hemostatic activation. Anesth Analg 1997; 85: 498-506. 